\title{
Rational strain selection and engineering creates a broad-spectrum, systemically effective oncolytic poxvirus, JX-963
}

\author{
Steve H. Thorne, ${ }^{1,2}$ Tae-Ho H. Hwang, ${ }^{3}$ William E. O'Gorman, ${ }^{4}$ David L. Bartlett, ${ }^{5}$ Shizuko Sei, ${ }^{6}$ \\ Femina Kanji, ${ }^{7}$ Christopher Brown, ${ }^{8}$ Joel Werier, ${ }^{9}$ Jin-Han Cho, ${ }^{10}$ Dong-Ewon Lee, ${ }^{11}$ \\ Yaohe Wang, ${ }^{12}$ John Bell, ${ }^{2,7}$ and David H. Kirn² \\ 1Department of Pediatrics and Bio-X Program, James H. Clark Center, Stanford University School of Medicine, Stanford, California, USA. \\ 2Jennerex Biotherapeutics, San Francisco, California, USA. ${ }^{3}$ Department of Pharmacology, Medical College of Dong-A University, Busan, \\ Republic of Korea. ${ }^{4}$ Department of Microbiology and Immunology, Stanford University School of Medicine, Stanford, California, USA. \\ ${ }^{5}$ Division of Surgical Oncology, Department of Surgery, University of Pittsburgh, Pittsburgh, Pennsylvania, USA. ${ }^{6}$ Viral Vector Toxicology Section, \\ LHTP, SAIC-Frederick Inc., National Cancer Institute-Frederick, Frederick, Maryland, USA. ${ }^{7}$ Ottawa Health Research Institute, Ottawa, Ontario, Canada. \\ ${ }^{8}$ Biochemistry, Microbiology and Immunology Department, University of Ottawa, Ottawa, Ontario, Canada. ${ }^{9}$ Ottawa General Hospital, Ottawa, Ontario, Canada. \\ ${ }^{10}$ Department of Radiology and ${ }^{11}$ Department of Pharmacology, Medical College of Dong-A University, Busan, Republic of Korea. \\ ${ }^{12}$ Barts and the London School of Medicine and Dentistry, Queen Mary's University of London, London, United Kingdom.
}

\begin{abstract}
Replication-selective oncolytic viruses (virotherapeutics) are being developed as novel cancer therapies with unique mechanisms of action, but limitations in i.v. delivery to tumors and systemic efficacy have highlighted the need for improved agents for this therapeutic class to realize its potential. Here we describe the rational, stepwise design and evaluation of a systemically effective virotherapeutic (JX-963). We first identified a highly potent poxvirus strain that also trafficked efficiently to human tumors after i.v. administration. This strain was then engineered to target cancer cells with activation of the transcription factor E2F and the EGFR pathway by deletion of the thymidine kinase and vaccinia growth factor genes. For induction of tumor-specific cytotoxic T lymphocytes, we further engineered the virus to express human GM-CSF. JX-963 was more potent than the previously used virotherapeutic Onyx-015 adenovirus and as potent as wild-type vaccinia in all cancer cell lines tested. Significant cancer selectivity of JX-963 was demonstrated in vitro in human tumor cell lines, in vivo in tumor-bearing rabbits, and in primary human surgical samples ex vivo. Intravenous administration led to systemic efficacy against both primary carcinomas and widespread organ-based metastases in immunocompetent mice and rabbits. JX-963 therefore holds promise as a rationally designed, targeted virotherapeutic for the systemic treatment of cancer in humans and warrants clinical testing.
\end{abstract}

\section{Introduction}

Targeted therapies hold great promise for the treatment of cancer, but novel agents are still needed, as resistance frequently develops through mutation of the target molecules and/or tumor escape through pathway redundancies. Oncolytic viruses (virotherapeutics) are replication-competent, therapeutic biological agents whose replication is restricted to malignant cell types, either inherently or through genetic engineering (1). Selective intratumoral replication leads to virus multiplication, killing of the infected cancer cell by unique and apoptosis-independent mechanisms (oncolysis), and spread of the virus to other tumor cells. Virotherapeutics therefore have the potential to effectively treat refractory cancers, and clinical proof of concept has been achieved with local administration for several oncolytic viruses (2). However, for oncolytic viruses to have a major impact on patient survival, systemic efficacy and i.v. delivery will be needed.

We therefore undertook a stepwise design and development strategy to create a more effective systemic agent. First, we identi-

Nonstandard abbreviations used: Ad5, adenovirus serotype 5; TK, thymidine kinase; VGF, vaccinia growth factor; vvDD, vaccinia virus with double deletion of viral $T K$ and $V G F$ genes; WR, Western Reserve strain of vaccinia.

Conflict of interest: The authors have declared that no conflict of interest exists. Citation for this article: J. Clin. Invest. 117:3350-3358 (2007). doi:10.1172/JCI32727. fied poxviruses such as vaccinia as a virus species that has evolved for systemic dissemination and resistance to clearance by complement and antibodies $(3,4)$. We proceeded to select a vaccinia strain and selected and defined the mechanism of tumor targeting for gene deletions that optimized the safety, efficacy, and systemic deliverability of the virus. Finally GM-CSF was expressed from within the virus to maximize its tumor-killing potential by providing the virus with multiple mechanisms of action. The resulting virus (JX-963; Western Reserve strain of vaccinia [WR] with deletions in the viral thymidine kinase $[T K]$ and vaccinia growth factor $[V G F]$ genes and expressing human GM-CSF) was found to be safe and effective following systemic delivery in a variety of preclinical tumor models.

\section{Results}

Evaluation of poxvirus strains as systemic oncolytic therapeutics. A panel of vaccinia viruses used during the vaccination program and some related laboratory strains were screened for their ability to replicate in normal (normal human bronchial epithelial [NHBE] or small airway bronchial epithelial [SAEC]) and tumor (A2780 or HCT 116) cells. All vaccinia strains replicated to higher levels in the tumor cell lines than in the normal cells, indicating an inherent tumor selectivity for vaccinia strains (Figure 1A), but the therapeutic index (tumor to normal cell replication ratio) varied 

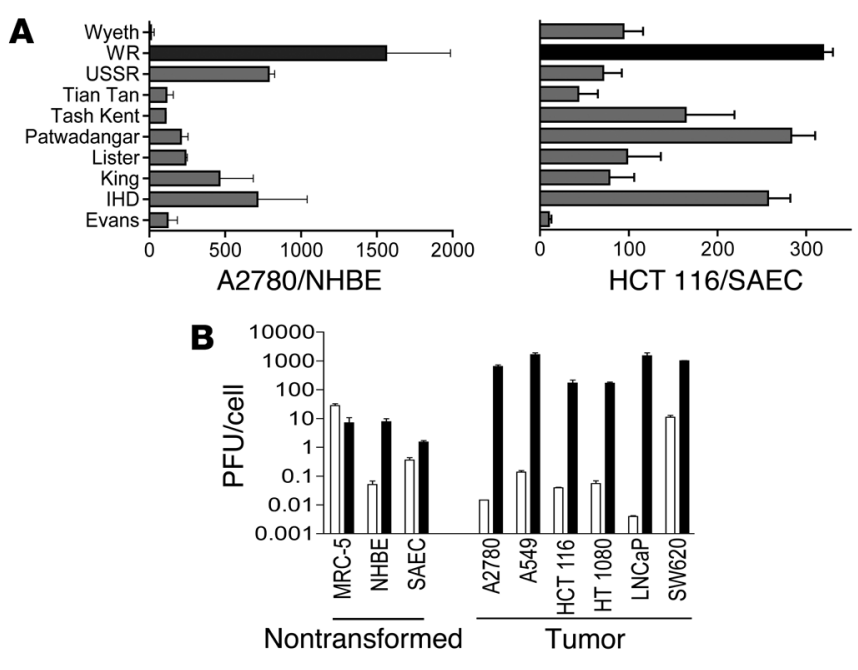

between strains. Strains used extensively in the laboratory are likely to have developed reduced pathogenicity in vivo and/or improved replication in transformed cells (due to their continual passage in these cell lines), and it was found that these strains (such as WR) tended to display greater inherent tumor selectivity in vitro than their parental vaccine strains (e.g., Wyeth), primarily due to enhanced replication in the tumor cell lines. This observation of inherent tumor selectivity of WR was extended in a panel of tumor and normal cell lines (Figure 1B) and represents the first time to our knowledge that wild-type vaccinia strains have been shown to display inherently superior replication in tumor cells relative to normal cells. This is not true for all viruses, however, as adenovirus serotype 5 (Ad5) (the backbone for the majority of oncolytic viruses in the clinic) did not display such selectivity.

Strain WR was selected for further examination because it demonstrated superior potency against multiple cancer cell lines and the largest therapeutic index of the strains screened (Figure 1). WR also replicated significantly better than Ad5 in multiple cancer cell lines $(P<0.05)$ but not in normal or nontransformed cells. The major limitation of most oncolytic viruses tested to date is an inability to efficiently infect tumors following systemic delivery, as seen when $1 \times 10^{9} \mathrm{PFU}$ of Ad5 was delivered i.v. to subcutaneous tumor models in mice (Figure 2); this equates to a dose of $3.5 \times 10^{12} \mathrm{PFU}$ in a $70-\mathrm{kg}$ human, higher than has ever been given to a patient.

\section{Figure 2}

Systemic delivery of viral strains to tumors. $1 \times 10^{9} \mathrm{PFU}$ of vaccinia strain WR or Ad5 were delivered i.v. to immunocompetent mice bearing subcutaneous CMT 64 or JC tumors (both lines susceptible to replication by both viruses after intratumoral injection). Mice were sacrificed after 48 or 72 hours or after 10 days and immunohistochemistry performed against viral coat proteins on paraffin-embedded sections of tumor tissue. (A) Graphs show scoring of positive cells in each tumor (asterisk indicates none detectable). For each condition, results are based on tumors from 3 mice, and for each tumor, 10 randomly chosen fields of view were scored. (B) Representative photographs show sections at 72 hours and 10 days after treatment (original magnification, $\times 100$ ).

\section{Figure 1}

Virus species and strain selection. (A) Burst ratio of vaccinia strains in tumor relative to normal cells. Different vaccinia strains were used to infect both primary normal cells (normal human bronchial epithelial [NHBE] or small airway bronchial epithelial [SAEC]) and tumor cell lines (A2780 or HCT 116) at an MOI of 1.0 PFU/cell. Virus collected 48 hours later was titered by plaque assay, and the ratio of virus produced (per cell) in tumor relative to normal cells is represented. (B) Different cell lines were infected with either WR (black bars) or Ad5 (white bars) at an MOI of 1.0 PFU/cell. Amounts of virus produced (infectious units/cell) 48 hours later were titered by plaque assay (left grouping: nontransformed and primary cells; right grouping: tumor cell lines).

Little or no replicating virus was evident in tumors (as detected by immunohistochemical staining for viral coat proteins 48 and 72 hours after viral delivery; susceptibility of these tumor cells for Ad5 replication following intratumoral injection has been demonstrated; ref. 5). Vaccinia strain WR, however, could effectively traffic to and infect the tumors in these same models, with up to $50 \%$ of the tumor cells staining positive within 48 hours of treatment. Furthermore, vaccinia was able to persist in the tumor for at least 10 days (Figure 2B), despite the fact that an antiviral immune response was initiated by this time.

Engineering of optimal cancer selectivity of vaccinia strain via double gene deletion. In order to maximize safety, particularly for i.v. administration in potentially immunodeficient cancer patients, attenuating and tumor-targeting genetic deletions were introduced into the virus. We have previously described preferential tumor expression
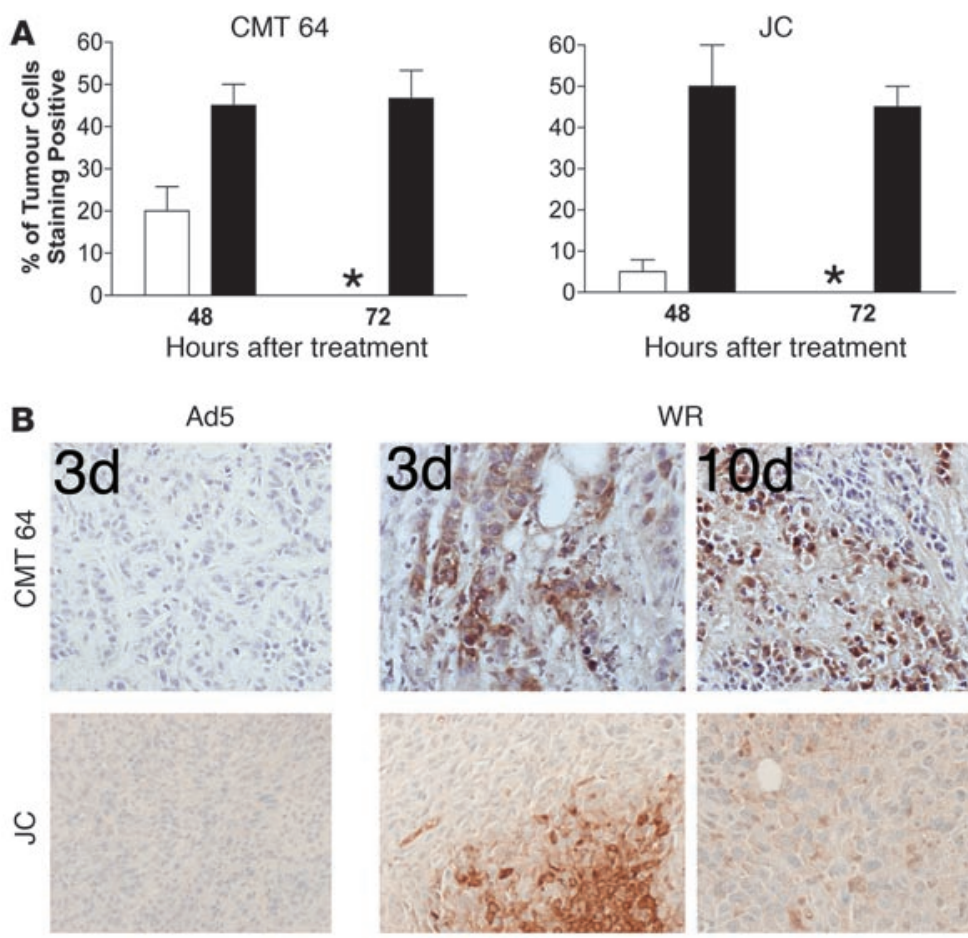

WR

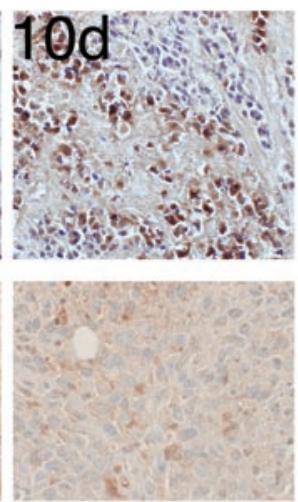




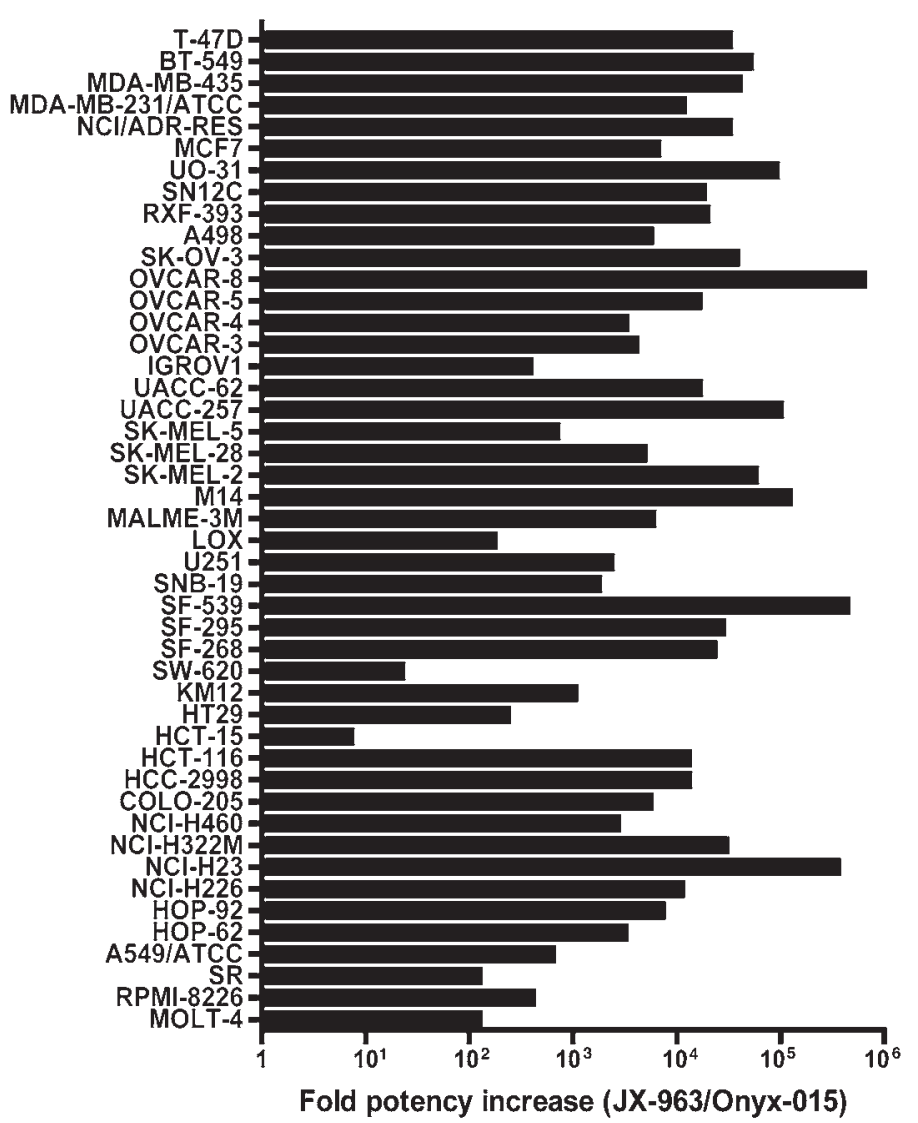

of transgenes from vvDD, a vaccinia virus with deletions in both the $T K$ and vaccinia growth factor $(V G F)$ genes $(6,7)$. Replication of this virus would be expected to be restricted to cells with elevated E2F levels (as E2F drives production of the cellular TK gene product; ref. 8) and/or activation of the EGFR pathway (as activation of this pathway by VGF is necessary for efficient viral replication; ref. 9).

However, despite the fact that both TK and VGF single deletions have previously been shown to attenuate the pathogenicity of the virus in vivo $(10,11)$, the double-deleted virus, $\mathrm{vvDD}$, was found to be capable of destroying tumor cell lines in vitro as efficiently as the parental WR strain (Supplemental Figure 1; supplemental material available online with this article; doi:10.1172/JCI32727DS1). This was true for a panel of more than 60 tumor cell lines, from 9 different organs of origin, with never more than a 2 -fold reduction in potency. Another desirable attribute for an oncolytic agent is rapid intratumoral spread (12). This can be achieved through a short replication cycle and early release of virus from infected cells. The $\mathrm{EC}_{50}$ values (number of viral PFU required to reduce the viability of a cell layer by $50 \%$ ) at 72 hours after infection were less than 0.2 for all but 4 of 55 nonhematopoietic cancer cell lines tested, highlighting the rapid cancer cell killing achieved with only small initial doses of double-deleted vaccinia virus.

We further found that the TK and VGF double-deleted (vvDD) backbone displayed markedly greater potency compared with the oncolytic adenovirus strain $d l 1520$ (Onyx-015) (13) (Figure 3). The vaccinia strain was approximately 10 -fold to 100,000 -fold more potent than Onyx-015, despite the fact that loss of tumor cell viability was evaluated 3 days after addition of vvDD but 6 days after addition of Onyx-015.

\section{Figure 3}

Cytopathic effect of JX-963 (vvDD expressing GM-CSF) versus Onyx-015 on a panel of human tumor cell lines. $\mathrm{EC}_{50}$ values were determined 3 days following infection of tumor cell lines with JX963 and 6 days after Onyx-015 infection. The ratio of the Onyx$015 \mathrm{EC}_{50}$ to the JX-963 $\mathrm{EC}_{50}$ was plotted (a value greater than 1.0 indicates that JX-963 was more potent).

Because the TK and VGF deletions produced little or no reduction in tumor cell killing relative to the wild-type strain and up to an 100,000-fold increase in potency over Onyx-015, we evaluated the effect of these deletions on viral replication in normal tissues and defined the mechanism of tumor selectivity. As mutation of the viral TK gene will create a dependence on cellular TK for successful viral replication, viral replication should correspond to cellular TK levels for TK-deleted vaccinia strains. Cellular TK levels are increased in the S-phase of the cell cycle of normal cells and constitutively in cancer cells (8); therefore, we examined the cellular TK levels and the viral replication and gene expression of different viruses in different cells and under different conditions. In addition, the viral VGF gene product is known to bind to the ErbB-1 (EGFR) protein and trigger a signaling pathway that includes phosphorylation of the ERK protein and results in cellular proliferation (14) (and thus increases in cellular TK levels). The levels of phosphorylated ERK (pERK) were therefore also examined. It was initially found that, as expected, cellular TK levels were high in nontransformed (Beas-2B) cells during proliferation, but that these levels were reduced after serum starvation. In tumor cells (HCT 116), however, serum starvation only produced a minor reduction in cellular TK levels (Figure 4A). In addition, the levels of pERK were also far more dramatically reduced upon serum starvation in the nontransformed compared with the transformed cell lines. Activation of the EGFR signaling pathway in serum-starved Beas-2B cells (through addition of either EGF or VGF protein) resulted in upregulation of $\mathrm{pERK}$ and a small increase in levels of cellular TK (Figure 4A). In contrast, levels of pERK and TK were not reduced by serum starvation in the HCT 116 tumor cells, and so subsequent addition of VGF or EGF had no effect (data not shown). Because vvDD displayed no alteration in its ability to initially infect cells relative to WR (data not shown), we examined whether the activation status ( $\mathrm{pERK}$ and TK levels) of a cell prior to infection affected early steps in the viral replication cycle. This was evaluated by quantifying viral expression of the luciferase reporter gene at early time points (4 hours) after infection for different viral strains (Figure 4B). It was first found that WR gene expression was unaffected by serum starvation, but that VGF or EGF pretreatment of serum-starved Beas-2B cells resulted in greater viral gene expression than was produced even by proliferating cells. This indicates for the first time to our knowledge that VGF is not only important in autocrine activation of signaling pathways in infected cells but plays a perhaps even more important role in "priming" neighboring cells for subsequent viral infection. As a result, serum-starved Beas$2 \mathrm{~B}$ cells preincubated with VGF and infected with TK-deleted virus displayed viral gene expression equivalent to that of cells infected with wild-type virus. Therefore, the TK deletion by itself may be insufficient under certain circumstances to create a reduction in viral replication in normal cells. Combination of TK and VGF viral deletions therefore is likely to create a more tumor-specific virus. 
A
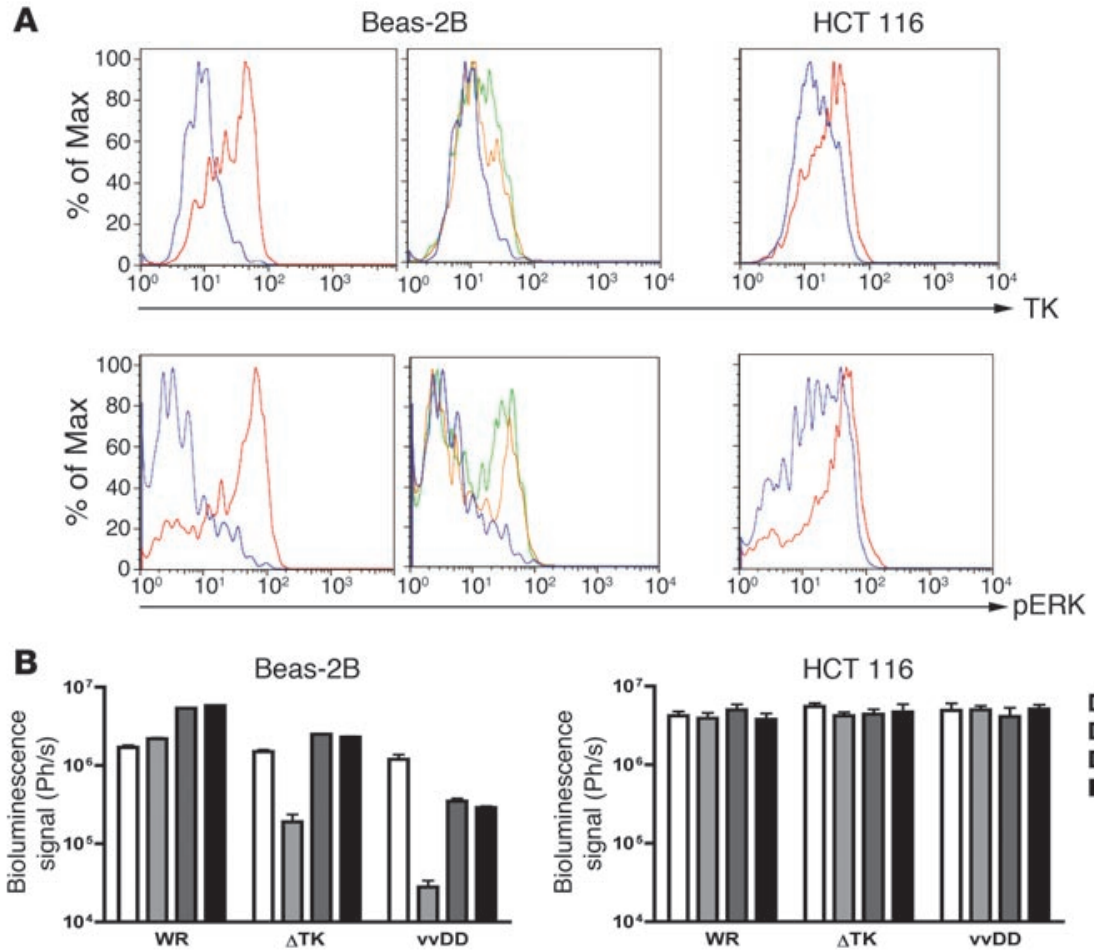

Figure 4

Mechanisms of selectivity of vvDD for tumor cells. (A) Levels of pERK and TK within cells under different conditions. Cell lines (Beas-2B, nontransformed; and HCT 116, transformed) were grown overnight in media with serum (red); without serum (blue); without serum and with EGF added 30 minutes before sampling (green); or serum starved with VGF added 30 minutes before sampling (yellow). Cells were then fixed and permeabilized before staining for $\mathrm{pERK}$ or TK and analyzed by flow cytometry ( $y$ axes are percent of maximum). (B) Viral gene expression early after infection of cells under different conditions. Cells grown as above were infected with vaccinia strains (WR; WR with TK deletion [ $\Delta \mathrm{TK}]$; vvDD) expressing luciferase at an MOI of 1.0. Luciferase levels were measured by bioluminescence imaging 4 hours after infection.

Indeed, of all the viruses, the double-deleted virus (vvDD) displayed the greatest reduction in gene expression in the serum-starved Beas$2 \mathrm{~B}$ cells, and this was only partially restored by addition of VGF (presumably due to the requirement for constant VGF exposure to maintain cells in a state of activated EGFR signaling pathway; ref. 14). All viruses produced equivalent gene expression following infection of the HCT 116 cells, under all the conditions examined.

It was further determined whether viral infection subsequently altered the cellular levels of pERK. It was seen (Figure 5A) that in serum-starved Beas-2B cells, WR was able to upregulate pERK levels 4 hours after infection, while vvDD was not. This corresponded with the subsequent ability of these viruses to successfully replicate in these cells (Figure 5B), and so it appears the attenuation of vvDD in normal cells is likely related to an inability to induce EGFR signaling pathways to compensate for the requirement for cellular TK. In HCT 116 cells, serum starvation did not significantly reduce pERK levels, and so the fact vvDD is unable to phosphorylate this protein did not affect its ability to replicate in this cell line. It was also found that whereas single (TK or VGF) or double deletions did not reduce viral replication in tumor cell lines, a reduction of between 1 and 2 logs was seen in nonproliferating, nontransformed cells (Figure 5B). Because of the natural tumor selectivity of the WR strain of vaccinia virus (Figure 1B), this results in a total therapeutic index (reduction of viral replication lial, bone marrow, or ovarian cells). In vivo viral gene expression was studied by noninvasive bioluminescence imaging, and viral biodistribution was examined postmortem in immunocompetent tumor-bearing mice (Figure 6). Bioluminescence imaging following i.v. delivery of $1 \times 10^{7} \mathrm{PFU}$ of WR or vvDD expressing luciferase showed that the viruses displayed similar initial infection and viral gene expression patterns (including in spleen, lung, liver, and tumor) (Figure 6A). However, the bioluminescence signal from vvDD was rapidly cleared from most organs other than the tumor, even in the immunodeficient mice, while WR continued to replicate in the target organs and spread to other tissues, including bone marrow, skin, and brain (Supplemental Figure 2). Although vvDD did produce some points of infection outside of the tumor at late time points, these appeared transiently, indicating secondary spread from infected tumors without replication in normal tissue (Supplemental Figure 3); postmortem analysis indicated this occurred primarily in the skin (data not shown). Recovery of infectious viral units from tissues of immunocompetent mice treated i.v. with $1 \times 10^{9} \mathrm{PFU}$ of vvDD (a lethal dose for WR) revealed that by day 8 after treatment, the tumor displayed increasing viral titer, with over 1,000 -fold more viral copies per milligram tissue than any other organ, while all normal tissues were below the limits of detection or showed falling viral titers (Figure 6C). No significant toxicity was demonstrated even at 
A

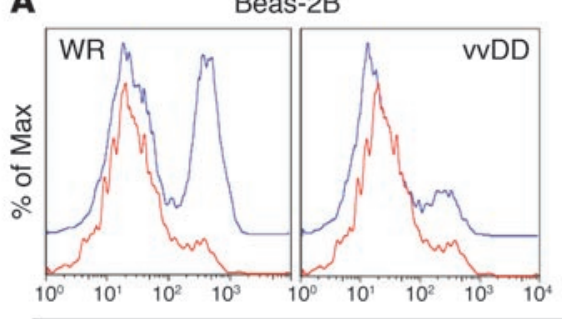

HCT 116

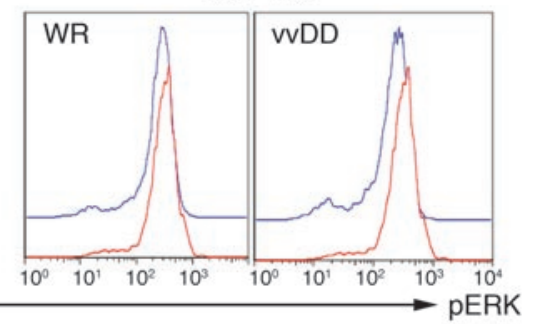

B

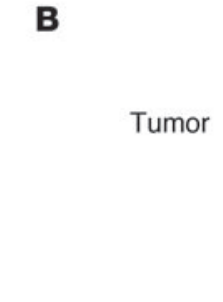

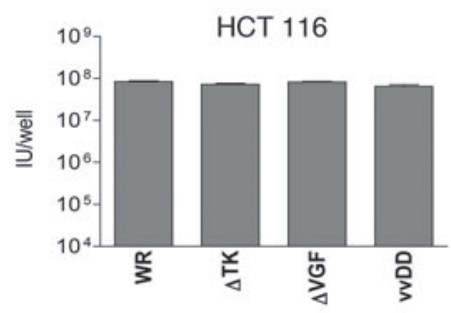

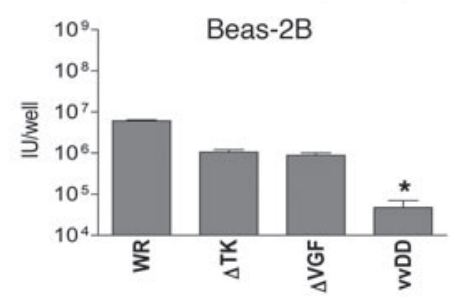

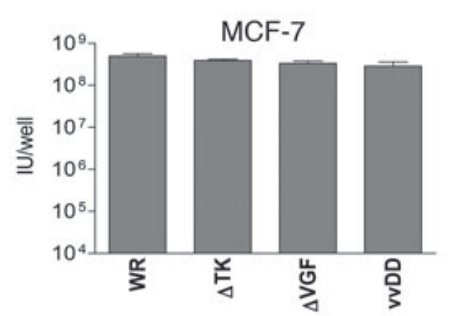

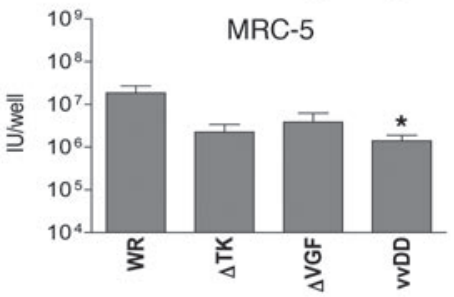

\section{Figure 5}

Selectivity and potency of vaccinia deletion mutants in vitro. (A) Beas-2B (nontransformed) and HCT 116 (tumor) human cells were grown overnight in media without serum and infected with an MOI of 1.0 of viruses WR or VVDD. Cells were sampled 0 (red) or 4 hours (blue) after infection, stained for $\mathrm{pERK}$, and assayed by flow cytometry. (B) Human tumor cell lines (HCT 116 and MCF-7) or human immortalized but nontransformed cell lines (Beas-2B and MRC-5), grown serum starved overnight, were treated with different strains of vaccinia at an $\mathrm{MOI}$ of 1.0 PFU/cell. Strains used were WR and WR containing deletions in the TK gene $(\Delta T K)$, the VGF gene ( $\triangle V G F)$, or both of these genes (VvDD). Virus produced after 48 hours was titered by plaque assay. Asterisks indicate vvDD replication significantly reduced relative to WR, $P<0.05$. these high i.v. doses. In order to verify whether viral replication and subsequent shedding into the blood after i.v. administration was tumor dependent, we administered i.v. doses of the vvDD backbone to identical immunocompetent mice with and without tumors. A secondary wave of viremia was found in tumor-bearing animals (with $28 \pm 12$ viral genomes/ $\mu \mathrm{g}$ wbc DNA detected 3 days after treatment) consistent with intratumoral genome replication (non-tumor-bearing animals displayed less than 2 genomes/ $\mu \mathrm{g}$ wbc DNA at this time point) (Supplemental Figure 4).

Further confirmation of the tumor selectivity of viral replication and gene expression from the TK and VGF double-deleted viral backbone (vvDD) was witnessed in tumor and normal tissue explants (Supplemental Figure 5). A panel of 6 primary human tissue specimens and, where available, adjacent normal tissue samples, were infected with vvDD expressing GFP. Viral gene expression (GFP fluorescence) imaging 24 hours after infection indicated high levels of viral replication in all 6 of the tumor specimens, but there was no detectable fluorescence in the normal (rectal, liver, or muscle) tissue adjacent to the tumor.

Antitumor efficacy of i.v. vvDD in immunocompetent murine and buman tumor xenograft models. The antitumor effects of vVDD were then analyzed in immunocompetent mouse tumor models and in human tumor xenograft models. vvDD was first compared with Wyeth TK-deleted vaccinia (as this is the most common vaccinia backbone used in clinical trials) in order to compare the viral backbones (as human GM-CSF is not active in rodents, only the oncolytic potential of the viral backbone was compared in these models). vvDD had significantly greater antitumor effects than the Wyeth TK-deleted vaccinia strain $(P=0.04)$ when both were delivered i.v. (Figure 7A). Further studies showed that $1 \times 10^{9} \mathrm{PFU}$ of vvDD was capable of significant antitumor effects when delivered either systemically (i.p. route leading to i.v. dissemination) or by intratumor- al injection to both immunodeficient mice carrying human tumor xenografts and immunocompetent mice bearing syngeneic tumors (Figure 7B). This efficacy in murine tumor models is despite the fact that vaccinia virus replication and cytotoxicity are markedly lower in murine than human cancer cells (data not shown).

$J X-963$ (vvDD-GM-CSF) final product engineering and evaluation in immunocompetent rabbit tumor model: i.v. efficacy against established systemic, organ-based tumor metastases. In order to increase the antitumor potential of vvDD, and to suppress the outgrowth of microscopic tumor deposits that are not vascularized at the time of i.v. dosing, the cytokine GM-CSF was inserted into the site of the TK gene (under the control of the synthetic early/late promoter; ref. 15); this virus was designated JX-963. Because human GM-CSF is not active in rodents but is active in rabbits (16), and in order to assess the activity against much larger primary tumors that reproducibly metastasize, JX-963 was used in a rabbit model with primary (VX2) liver tumors and lung metastases (17). At the time of dosing, multiple 1- to 2-mm tumor metastases were already evident on histopathology of the lungs of control rabbits. JX-963 was compared with vvDD (to assess the potential of GM-CSF expression to improve the benefits of the vvDD viral backbone) and with JX-594 (Wyeth TK-deleted vaccinia expressing GM-CSF; in order to assess any benefits of expressing GM-CSF from vvDD relative to the traditionally used Wyeth strain). As in the mouse models, $1 \times 10^{9}$ PFU of i.v. vvDD had significant antitumor effects (Figure 8). The $\mathrm{vvDD}$ virus was also capable of inhibiting the outgrowth of microscopic lung metastases. In order to assess additional efficacy due to concomitant GM-CSF expression, JX-963 was compared directly with vvDD. JX-963 produced greater efficacy against the primary tumor and completely blocked the outgrowth of lung metastases. Furthermore, JX-963 was compared with JX-594 in order to assess the vvDD oncolytic backbone relative to TK-deleted Wyeth for the 

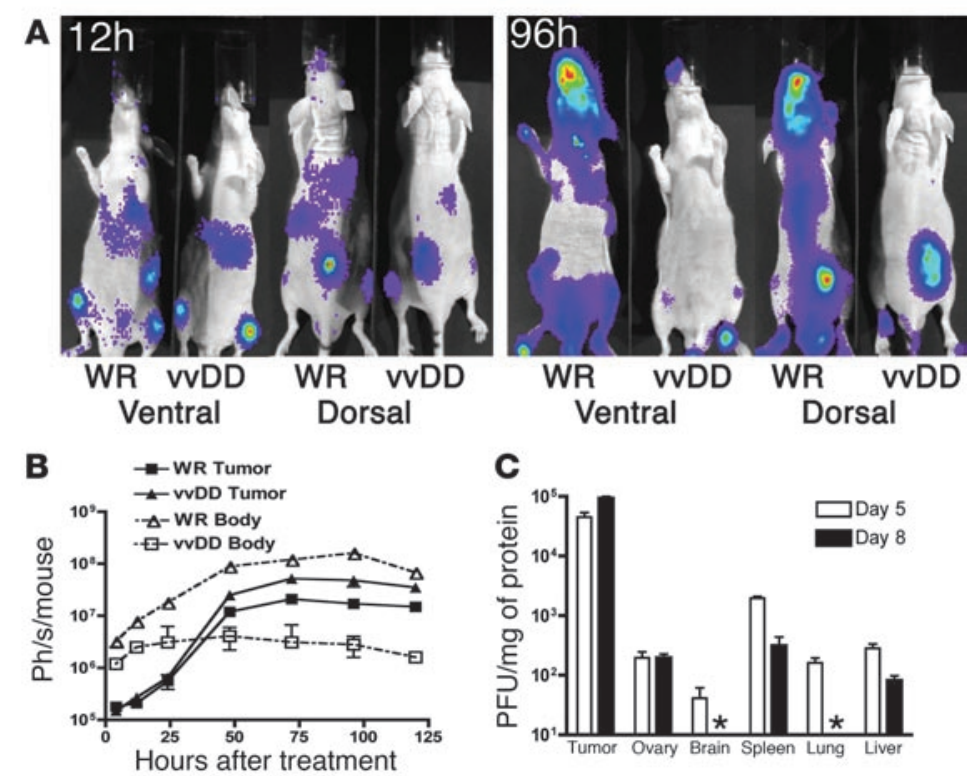

\section{Figure 6}

Intravenous delivery and tumor selectivity of vvDD versus wild-type vaccinia (WR). (A) Biodistribution of WR and VVDD following systemic delivery to tumor-bearing mice. Athymic CD1 nu/nu mice bearing subcutaneous human HCT 116 tumors (arrows) were treated with $1 \times 10^{7}$ PFU of vaccinia strains via tail vein injection. Viral strains (WR and vVDD) expressed luciferase, and the subsequent biodistribution of viral gene expression was detected by bioluminescence imaging in an IVIS 100 system (Xenogen; Caliper Life Sciences) following addition of the substrate luciferin at the times indicated after treatment. Representative mice from $n=5$ /group are shown; the remaining mice are shown in Supplemental Figure 6. (B) Viral gene expression, as quantified by light production, was plotted over time for the regions of interest covering the whole body (ventral image) (dashed line, open symbols) or from the tumor only (dorsal view, region of interest over tumor) (solid line, filled symbols) for BALB/c mice bearing subcutaneous JC tumors ( $n=5$ mice/group) and treated with $1 \times 10^{7}$ PFU of either virus by tail vein injection. (C) Recovery of vVDD delivered systemically (i.p. injection of $1 \times 10^{9} \mathrm{PFU}$ ) to C57BL/ 6 mice bearing subcutaneous MC38 tumors. Mice were sacrificed on days 5 or 8 after treatment ( $n=8 /$ group), different tissues recovered, and viral infectious units (PFU/mg tissue) titered by plaque assay (asterisk indicates below the limits of detection).

expression of GM-CSF. Again, JX-963 produced greater benefits against both the primary tumor and metastases. Human GM-CSF was detected in the plasma of both JX-963- $(610 \mathrm{pg} / \mathrm{ml}$ at day 4$)$ and JX-594-treated $(180 \mathrm{pg} / \mathrm{ml})$ tumor-bearing rabbits but not in tumor-free rabbits (Supplemental Figure 6). This increased GMCSF production with JX-963 relative to JX-594 is likely to be correlated to increased viral replication, as infectious viral units shed into the blood in this model indicated that vvDD backbone viruses replicated to a much greater degree than the Wyeth backbone, which is expected, as WR is a more pathogenic strain of vaccinia than the Wyeth strain (Figure 8C). This additional replication is almost certainly limited to the tumor, however, as vvDD delivered to non-tumor-bearing rabbits produced no secondary shedding of virus into the blood. It was also observed that vvDD produced even greater viral replication than JX-963, possibly indicating that GM-CSF expression may lead to earlier clearance of the virus; this was despite the much greater antitumor benefits elicited by JX-963. One likely reason for this is that, in addition to direct oncolytic effects, JX-963 was also found to cross-protect the ani- mal against the tumor by raising a CTL response against the VX2 tumor cells (Supplemental Figure 7) (unfortunately, however, a sample loss meant JX-963 could not be compared directly with vvDD in this assay).

\section{Discussion}

We selected vaccinia virus as a platform for the design of oncolytic viruses because of several favorable attributes: vaccinia has evolved well-defined mechanisms to spread through the blood to epidermal tissues without inactivation during its life cycle; poxviruses can spread extremely rapidly within tissues (motile local and distant spread is driven by actin tail formation on cell-associated enveloped virus [CEV] and extracellular enveloped virus [EEV] forms); the transgene-encoding capacity of vaccinia is large relative to that of viruses such as adenovirus, making expression of multiple therapeutic and imaging transgenes feasible; and vaccinia has a long history of human use during the smallpox eradication campaign (including a well-defined list of contraindications to vaccinia use and available antiviral agents such as vaccinia immunoglobulin [VIG] or cidofovir to treat adverse events).

Through screening of vaccinia strains for tumor-selective replication, we were able to identify an inherently tumor-selective virus capable of systemic tumor delivery with rapid oncolytic effects. In order to improve the safety of this virus while targeting the majority of solid cancers in humans, 2 deletions capable of increasing its therapeutic index were introduced and found to functionally target EGFR pathway activation, including mutant ras. This double-deleted virus (vvDD, containing viral TK and VGF deletions) was found to be as potent as the wild-type strain against a large panel of tumor cell lines and displayed increased potency relative to the Onyx- 015 oncolytic virus. However, in nontransformed cells, this virus backbone was highly attenuated, and in vivo biodistribution studies revealed a rapid removal of the virus from all nontumor tissues. Dramatic therapeutic effects against large primary tumors were demonstrated following systemic delivery.

Finally, because it is unlikely that all tumor cells will be infected, even following efficient systemic viral delivery, GM-CSF was expressed from this viral backbone. The addition of GM-CSF was found to increase the effectiveness of this virus against primary tumors, prevent the outgrowth of micrometastases, and produced an antitumor CTL response, while JX-963 also produced significantly enhanced antitumor effects compared with JX-594, indicating the benefits of expressing GM-CSF from the vvDD backbone. This indicates that this virus, JX-963, is capable of systemic delivery to tumors, where it rapidly and efficiently destroys tumor tissue, while sparing normal organs, and at the same time induces an immune response within the tumor that is capable of recognizing tumor antigens produced in situ.

One potential limitation of using vaccinia virus as an antitumor agent is that, even though systemic delivery to the tumor is initially possible in naive individuals, the immune response raised by prior exposure to the virus may inhibit the efficacy of subsequent treatment. Thus, 4 tumor-bearing rabbits that had initially responded to treatment with JX-963 but had tumor progression after 4 weeks off of therapy were retreated with $1 \times 10^{9} \mathrm{PFU}$ of JX-963 delivered i.v. (Supplemental Figure 8). Preliminary results 


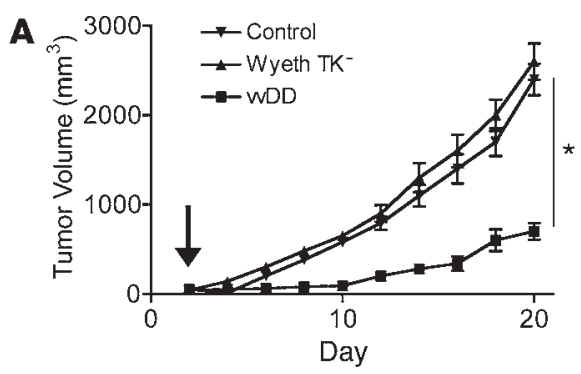

B

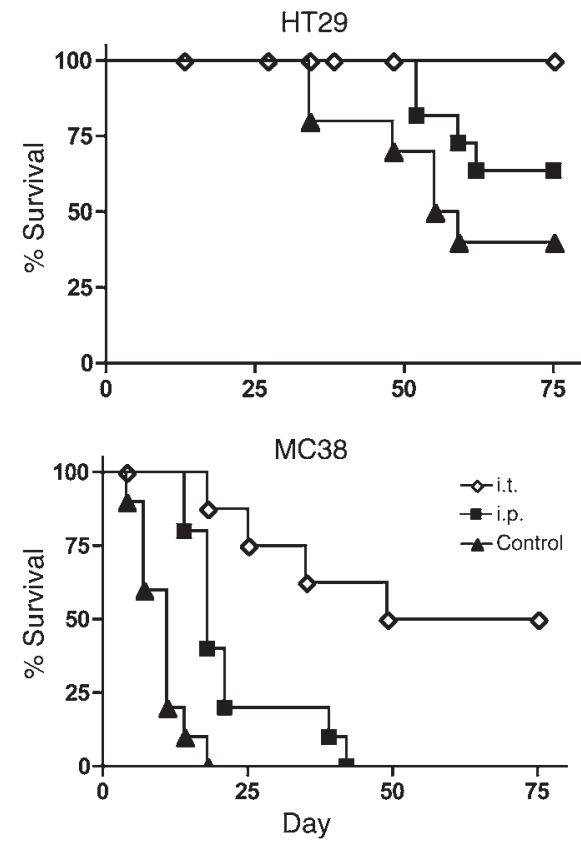

showed that this repeat treatment ( 6 weeks after the initial dose) resulted in a decrease in tumor size in 3 of the 4 animals. This was after a strong neutralizing antibody titer had been raised. Therefore, as may be predicted based on vaccinia biology in the blood, efficacy was feasible even in the presence of high-level neutralizing antibody titers. Future studies will explore the exact relationship between antibody titers and delivery to tumors i.v. and will determine whether these antitumor effects are caused by direct oncolysis or by boosting of an antitumor immune response.

JX-963 therefore was found to display rapid and potent antitumor effects, despite a high degree of attenuation in nontumor tissues. It is capable of systemic delivery, resulting in complete reduction of tumor burden, even against large primary tumors and metastatic disease. The multiple mechanisms of tumor cell killing employed by JX-963 mean that resistance is less likely to develop than for other targeted therapies that recognize a single target or rely on apoptotic tumor cell killing, and initial results indicate that an antitumor effect is feasible in previously immunized individuals. JX-963 therefore has the potential to effectively treat a wide spectrum of disseminated human tumors.

\section{Methods}

Viruses and cell lines. The panel of wild-type poxvirus strains (Wyeth, WR, USSR, Tian Tan, Tash Kent, Patwadangar, Lister, King, IHD, and Evans) was kindly provided by Geoffrey L. Smith, Imperial College, London. Human Ad5 was obtained from ATCC and dl1520 from Arnold J. Berk,

\section{Figure 7}

Efficacy of vvDD following delivery by different routes to tumor-bearing mouse models. (A) Single i.v. injections of $1 \times 10^{9} \mathrm{PFU}$ of viral strain vVDD or vaccinia Wyeth strain bearing a TK deletion were delivered to immunocompetent mice bearing subcutaneous TIB-75 tumors (50-100 $\mathrm{mm}^{3}$ ) (3 days after implantation; arrow). Tumor volume was measured by calipers ( $n=8 /$ group). ${ }^{*} P=0.04$ for vvDD relative to Wyeth TK(B) $1 \times 10^{9}$ PFU of vvDD was delivered intratumorally (i.t.) or i.p. to SCID mice bearing subcutaneous HT29 tumors or BALB/c mice bearing subcutaneous MC38 tumors. Kaplan-Meier survival curves were compared with those for the PBS-injected control group ( $n=8 /$ group). Mice were euthanized when tumors reached $1.4 \mathrm{~cm}^{3} . P<0.05$ for all treatment groups relative to PBS.

UCLA, Los Angeles, California, USA (18). The strain of WR in which VGF is partially deleted (vSC20) was kindly provided by Bernard Moss, NIH (10). The TK insertion-deletion strain of WR (vJS6) and the TK-, VGF-doubledeleted strain of WR (vvDD) as well as the Wyeth TK-deleted strain expressing GM-CSF (JX-594) have been described previously $(7,17)$. The WR strain expressing firefly luciferase was kindly provided by Gary Luker (University of Michigan, Ann Arbor, Michigan, USA) (19).

Vaccinia strain JX-963 was constructed by recombination of a version of the pSC65 plasmid containing the E. coli gpt and human CSF2 (encoding GM-CSF) genes (under the control of the p7.5 and PSE/L promoters, respectively) into the TK gene of the vSC20 strain of WR. Further selection of white plaques after propagation of the virus in $\mathrm{X}$-gal produced a virus with nonfunctioning lacZ (lacZ is expressed from within VGF in vSC20). Correct insertion into the TK gene and loss of lac $Z$ function were verified by sequencing and GM-CSF production verified by ELISA.

The wDD expressing luciferase was constructed by insertion of a version of the pSC65 plasmid with luciferase under control of the p7.5 promoter into vSC20. Bioluminescence was verified using an IVIS 50 system (Xenogen; Caliper Life Sciences).

The human tumor cell lines used in this study were A2780 (ovarian; obtained from ECACC), A549 (lung; ECACC), HCT 116, HT-29 and SW620 (colon; obtained from ATCC), HT-1080 (fibrosarcoma; ATCC), LNCaP (prostate; ATCC), PANC-1 (pancreatic; ATCC), MCF-7 (breast; ATCC). Nontransformed cells used were MRC-5 (lung fibroblast; ATCC), Beas-2B (bronchial epithelial; kindly provided by Tony Reid, UCSD, San Diego, California, USA) and the primary, normal cells (NHBE and SAEC), both obtained from Clonetics. In addition, the NCI-60 cell panel of cancer cell lines was used.

The mouse tumor cell lines used were CMT 64 (C57BL/6 NSLC; Cancer Research UK), JC (BALB/c mammary; ATCC), MC38 (C57BL/6 colon; NIH), and TIB-75 (BNL 1ME A.7R.1) (BALB/c hepatic; ATCC). The cell lines NIH $3 \mathrm{~T} 3$ and NIH $3 \mathrm{~T} 3$ with activated H-Ras were kindly provided by Richard Marais (Institute of Cancer Research, London, United Kingdom). The rabbit tumor cell line VX2 has been described previously (17).

In vitro replication, cytopathic effect, and viral gene expression assays. Cell lines were seeded into 6 -well plates at $5 \times 10^{6} \mathrm{cells} /$ well and left overnight. Virus was then added at an MOI of $1.0 \mathrm{PFU} /$ cell and allowed to infect for 2 hours. At the end of the infection, the media was changed and plates incubated for 48 hours; the cells were then scraped into the media and collected. Cells were lysed by 3 rounds of freezing and thawing followed by sonication, before serial dilutions of the crude viral lysate was added to BSC-1 cells to titer the virus by plaque assay. Adenovirus was titered by plaque assay on A549 cells. All experiments were run in triplicate.

In order to assess the cytopathic effect of the virus (Figure 1B), cells were seeded at 5,000 cells/well in 96-well plates and allowed to attach overnight. Serial dilutions of the viruses to be tested were then added to the plates in triplicate (MOI range from 100 to 0.001 ), and the plates incubated for a 

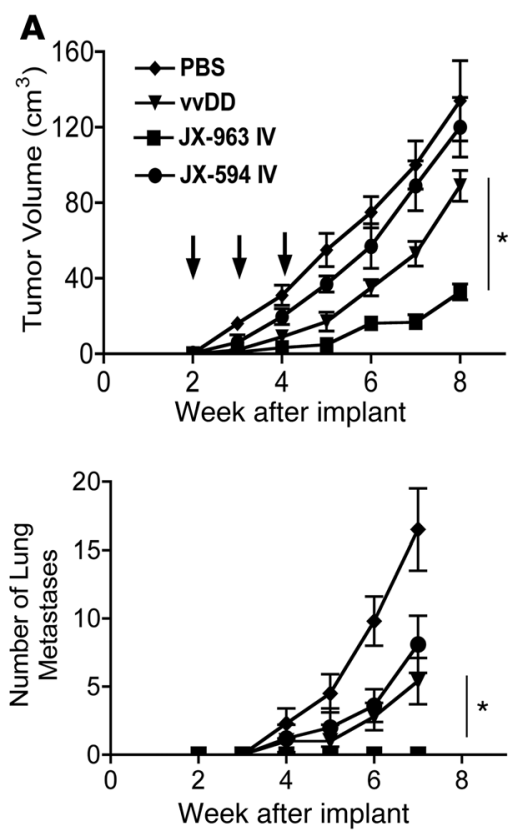

B

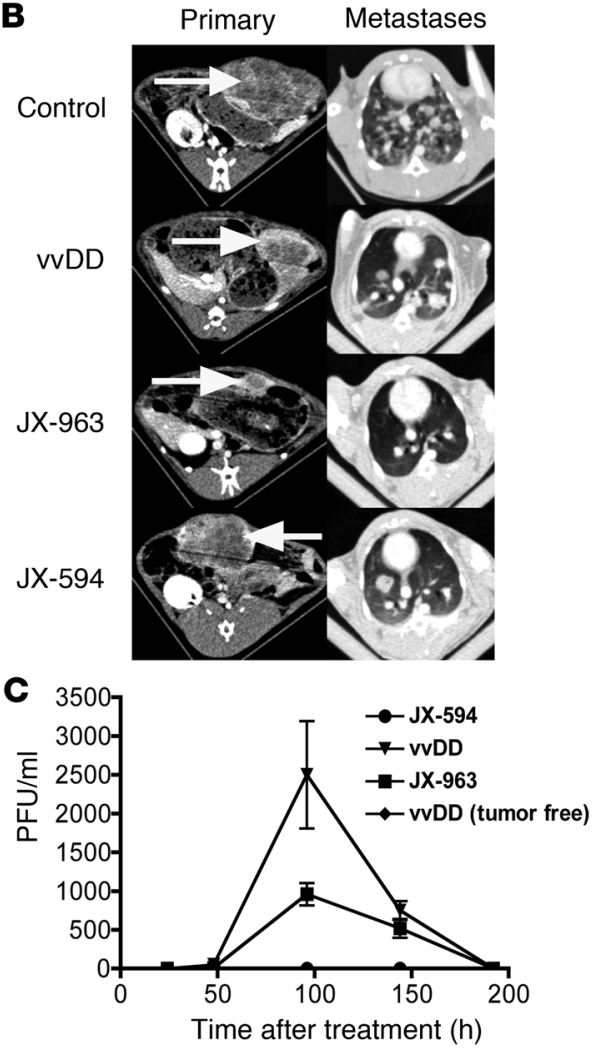

\section{Figure 8}

Rabbits bearing VX2 tumors implanted into the liver were followed by CT imaging at the indicated times after tumor implantation. (A) $1 \times 10^{8}$ PFU of viruses JX-594 (Wyeth strain, TK-deleted, expressing human GM-CSF), vvDD, or JX963 (vvDD-GM-CSF) were delivered by ear vein injection at 2, 3, and 4 weeks after tumor implantation (arrows), when tumors measured approximately $5 \mathrm{~cm}^{3}$. The primary tumor burden (top) and the number of detectable lung metastases (by CT scanning; bottom) were measured ( $n=18$ for control-treated animals; $n=6$ for vvDD-treated; $n=6$ for JX-963-treated) ${ }^{*} P<0.05$. (B) Representative CT scans of the primary tumor in the liver (left panels; tumor indicated by arrows) and metastases in the lungs (right panels) at 6 weeks after implantation. (C) Animals were also bled at the indicated times after treatment, and the numbers of viral infectious units $(\mathrm{PFU} / \mathrm{ml})$ were titered in the blood. further 72 hours. After this time, media was replaced with media without serum and MTS (Promega) added to the plates. After 2-4 hours incubation, the absorbance at $450 \mathrm{~nm}$ was read on an ELISA plate reader. Cytopathic effect was determined as reduction in cell viability of a test well relative to both untreated wells containing cells only (100\% viable) and cell-free wells ( $0 \%$ viable). Results were represented as the MOI at which $50 \%$ of the cell layer was viable $\left(\mathrm{ED}_{50}\right)$.

To evaluate the oncolytic spectrum of the vaccinia constructs (Figure 2 and Supplemental Figure 1), the NCI cancer cell line panel (http://dtp.nci. nih.gov/branches/btb/ivclsp.html) was employed as has been described for anticancer drug screens (20). The human tumor cell lines of the cell line panel were maintained as described previously (20) and seeded in 96-well microplates at plating densities ranging from 5,000 to 40,000 cells/well, depending on the doubling time of each cell line. The cells were incubated in duplicate with serially diluted WR or JX-963 inocula and examined for cell death at 72 hours after infection by XTT assay (21).

In some experiments, cells were grown with or without serum for 24 hours prior to infection; human EGF (Sigma-Aldrich) was added to serum-starved cells at a final concentration of $80 \mu \mathrm{g} / \mathrm{ml} 30$ minutes prior to viral addition. Alternatively, spent media from overnight cultures grown without serum and infected with vvDD (VGF-negative) or TK-deleted WR (VGF-positive) were filter sterilized through $0.2-\mu \mathrm{m}$ filters (to remove nonadherent cells and viral particles) and $1 \mathrm{ml}$ added per well to serum-starved cells 30 minutes prior to infection.

For bioluminescent imaging assays, virus expressing luciferase under control of the p7.5 early/late promoter was added to cells at an MOI of 1.0, and light production was measured 30 seconds after addition of luciferin $(300 \mu \mathrm{g} / \mathrm{ml})$ using an IVIS 50 system (Xenogen; Caliper Life Sciences).

Flow cytometry assays. Cells under various conditions were fixed, permeabilized, and stained for phospho-FACS as described previously (22). Briefly, media was aspirated and $1 \mathrm{ml}$ fresh medium added per well (in 6-well plates). $100 \mu 116 \%$ paraformaldehyde was added and cells scraped into the media. After 10 minutes, $2 \mathrm{ml}$ PBS plus $2 \%$ FBS was added to the cells and the cells spun and resuspended in 100\% methanol. After 10 minutes, cells were spun and resuspended in $100 \mu \mathrm{l}$ PBS plus $2 \%$ FBS. Cells were subsequently stained for phospho-p44/42 MAPK (Thr202/Tyr204) (Cell Signaling Technologies) and TK (Novus Biologicals) with appropriate secondary antibodies (BD Biosciences - Pharmingen), and cells were analyzed on a FACScaliber (BD Biosciences - Pharmingen).

Mouse syngeneic and xenograft tumor model studies. Immunocompetent mice were implanted subcutaneously with syngeneic tumor cells $\left(1 \times 10^{6}\right.$ cells/mouse), JC and TIB-75 cells were implanted into BALB/c mice, and MC38 and CMT 64 cells were implanted into C57BL/6 mice. Human xenograft models involved $1 \times 10^{7} \mathrm{HT} 29$ cells implanted subcutaneously into SCID mice (all mice were age $8-10$ weeks and sex matched). Once tumors reached $50-100 \mathrm{~mm}^{3}$, animals were regrouped and treated as indicated. Tumor sizes were followed by caliper measurement and animals euthanized when tumors reached $1.4 \mathrm{~cm}^{3}$. All animal studies were approved by Administrative Panel on Laboratory Animal Care (APLAC) committee, Stanford University; Institutional Animal Care and Use Committee (IACUC), University of Pittsburgh; or Institutional Animal Committee, Dong-A University.

Mice treated with luciferase-expressing virus were imaged using an IVIS 100 system (Xenogen; Caliper Life Sciences). Mice were injected i.p. with luciferin $(30 \mathrm{mg} / \mathrm{kg})$ and anesthetized (2\% isoflurane) prior to imaging.

Some mice were sacrificed at the times indicated after treatment, and organs were recovered for viral biodistribution or immunohistochemical studies. For viral biodistribution, organs were snap-frozen and ground before plaque assays were performed. For immunohistochemistry studies, organs were fixed in formalin before being embedded in paraffin blocks 
for sectioning. Sections were stained with $\mathrm{H} \& \mathrm{E}$ and for viral coat proteins (polyclonal anti-vaccinia antibody or polyclonal anti-hexon antibody for adenovirus-treated animals).

Rabbit model. The implantation of VX2 tumors into the livers of New Zealand white rabbits and the measurement of tumor progression and metastasis to the lungs by $\mathrm{CT}$ and ultrasound scans have been described previously (17).

Blood assays. Blood was collected from mice by mandibular bleeding and from rabbits by ear bleeds. White blood cells were collected by Ficoll extraction, or plasma was collected after centrifugation to remove cells. DNA was collected from wbc by extraction with Blood DNA collection kit (QIAGEN) for RT-PCR of viral genomes. RT-PCR was run as described previously (23). Plasma samples were used to determine circulating GM-CSF levels by ELISA (R\&D Systems). In addition, infectious viral units in whole blood were titered by plaque assay (see above) following 3 rounds of freezing/thawing of the blood to release virus.

CTL assay. CTL assay was performed by mixing labeled peripheral blood lymphocytes, obtained from rabbits treated as indicated, with VX2 tumor cells in different effector to target ratios. Cytotoxicity was determined by FACS using the ACT1 assay (Cell Technology).

Explant preparation, culture, and infection. Fresh tumor specimens were sliced into approximately $2-\mathrm{mm}^{3}$ pieces and then placed on a Surgifoam sponge that was presoaked with alpha medium containing 10\% FBS. Double-deleted vaccinia virus encoding GFP was then added directly to the specimen and allowed to infect for 90 minutes at $37^{\circ} \mathrm{C}$ before covering the sponge and specimen with medium containing serum. At 24-hour intervals, specimens were visualized using fluorescence microscopy.

Statistics. Kaplan-Meier curves were compared using the generalized Wilcoxon test. Tumor response rates and metastasis-free rates were compared with Fisher's exact test. Viral bursts were compared using 2-tailed Student's $t$ test. A $P$ value of less than 0.05 was considered significant.

\section{Acknowledgments}

We would like to thank Gary Luker for providing the WR strain expressing luciferase, Geoffrey L. Smith for providing several wildtype vaccinia strains, Bernard Moss for providing the VGF-deleted vaccinia strain, and Arnold J. Berk for providing adenovirus strain dl1520. The matched $3 \mathrm{~T} 3$ cells with activated H-Ras were kindly provided by Richard Marais, and the Beas-2B cells were provided by Tony Reid. B.H. Park (Dong-A University) helped with rabbit models; Quan-en Yang and Jodie Mussio provided technical assistance; and Joseph Tomaszewski and Robert Shoemaker (NCI-Frederick) provided critical advice and support. This project has been funded in whole or in part with federal funds from the National Cancer Institute, NIH, under contract N01-CO-12400. The content of this publication does not necessarily reflect the views of the Department of Health and Human Services, nor does mention of trade names, commercial products, or organizations imply endorsement by the US government. These studies were also partly supported by grants from the Korea Science and Engineering Foundation, through the Medical Research Center for Cancer Molecular Therapy at Dong-A University, Republic of Korea.

Received for publication May 18, 2007, and accepted in revised form August 15, 2007.

Address correspondence to: David H. Kirn, Jennerex Biotherapeutics, One Market Street, San Francisco, California 94105, USA. Phone: (415) 281-8886; Fax: (415) 598-2600; E-mail: dkirn@jennerex.com.

Steve H. Thorne's present address is: Division of Surgical Oncology, Department of Surgery, University of Pittsburgh, Pittsburgh, Pennsylvania, USA.
1. Thorne, S.H., Hermiston, T., and Kirn, D. 2005. Oncolytic virotherapy: approaches to tumor targeting and enhancing antitumor effects. Semin. Oncol. 32:537-548.

2. Parato, K.A., Senger, D., Forsyth, P.A., and Bell, J.C. 2005. Recent progress in the battle between oncolytic viruses and tumours. Nat. Rev. Cancer. 5:965-976.

3. Smith, G.L., Symons, J.A., Khanna, A., Vanderplasschen, A., and Alcami, A. 1997. Vaccinia virus immune evasion. Immunol. Rev. 159:137-154.

4. Buller, R.M., and Palumbo, G.J. 1991. Poxvirus pathogenesis. Microbiol. Rev. 55:80-122.

5. Hallden, G., et al. 2003. Novel immunocompetent murine tumor models for the assessment of replication-competent oncolytic adenovirus efficacy. Mol. Ther. 8:412-424.

6. Puhlmann, M., Gnant, M., Brown, C.K., Alexander, H.R., and Bartlett, D.L. 1999. Thymidine kinasedeleted vaccinia virus expressing purine nucleoside phosphorylase as a vector for tumor-directed gene therapy. Hum. Gene Ther. 10:649-657.

7. McCart, J.A., et al. 2001. Systemic cancer therapy with a tumor-selective vaccinia virus mutant lacking thymidine kinase and vaccinia growth factor genes. Cancer Res. 61:8751-8757.

8. Hengstschlager, M., et al. 1994. Different regulation of thymidine kinase during the cell cycle of normal versus DNA tumor virus-transformed cells. J. Biol. Chem. 269:13836-13842.

9. Andrade, A.A., et al. 2004. The vaccinia virus-stim- ulated mitogen-activated protein kinase (MAPK) pathway is required for virus multiplication. Biochem. J. 381:437-446.

10. Buller, R.M., Chakrabarti, S., Cooper, J.A., Twardzik, D.R., and Moss, B. 1988. Deletion of the vaccinia virus growth factor gene reduces virus virulence. J. Virol. 62:866-874.

11. Buller, R.M., Smith, G.L., Cremer, K., Notkins, A.L., and Moss, B. 1985. Decreased virulence of recombinant vaccinia virus expression vectors is associated with a thymidine kinase-negative phenotype. Nature. 317:813-815.

12. Wein, L.M., Wu, J.T., and Kirn, D.H. 2003. Validation and analysis of a mathematical model of a replication-competent oncolytic virus for cancer treatment: implications for virus design and delivery. Cancer Res. 63:1317-1324.

13. Heise, C., et al. 1997. ONYX-015, an E1B gene-attenuated adenovirus, causes tumor-specific cytolysis and antitumoral efficacy that can be augmented by standard chemotherapeutic agents. Nat. Med. 3:639-645.

14. Tzahar, E., et al. 1998. Pathogenic poxviruses reveal viral strategies to exploit the ErbB signaling network. EMBO J. 17:5948-5963.

15. Chakrabarti, S., Sisler, J.R., and Moss, B. 1997. Compact, synthetic, vaccinia virus early/late promoter for protein expression. Biotechniques. 23:1094-1097.

16. Cody, V., et al. 2005. Generation of dendritic cells from rabbit bone marrow mononuclear cell cultures supplemented with hGM-CSF and hIL-4. Vet.
Immunol. Immunopathol. 103:163-172.

17. Kim, J.H., et al. 2006. Systemic armed oncolytic and immunologic therapy for cancer with JX-594, a targeted poxvirus expressing GM-CSF. Mol. Ther. 14:361-370.

18. Barker, D.D., and Berk, A.J. 1987. Adenovirus proteins from both $\mathrm{E} 1 \mathrm{~B}$ reading frames are required for transformation of rodent cells by viral infection and DNA transfection. Virology. 156:107-121.

19. Luker, K.E., Hutchens, M., Schultz, T., Pekosz, A., and Luker, G.D. 2005. Bioluminescence imaging of vaccinia virus: effects of interferon on viral replication and spread. Virology. 341:284-300.

20. Monks, A., et al. 1991. Feasibility of a high-flux anticancer drug screen using a diverse panel of cultured human tumor cell lines. J. Natl. Cancer Inst. 83:757-766.

21. Weislow, O.S., et al. 1989. New soluble-formazan assay for HIV-1 cytopathic effects: application to high-flux screening of synthetic and natural products for AIDS-antiviral activity. J. Natl. Cancer Inst. 81:577-586.

22. Krutzik, P.O., and Nolan, G.P. 2006. Fluorescent cell barcoding in flow cytometry allows highthroughput drug screening and signaling profiling. Nat. Methods. 3:361-368.

23. Kulesh, D.A., et al. 2004. Smallpox and pan-orthopox virus detection by real-time 3'-minor groove binder TaqMan assays on the Roche LightCycler and the Cepheid smart Cycler platforms. J. Clin. Microbiol. 42:601-609. 